\title{
\begin{tabular}{l|l} 
POLITIQUES \& & Politiques et management public
\end{tabular}

\section{La comptabilité de gestion dans les villes : la nécessaire déconstruction d'un idéal-type}

Management accounting in towns and cities: the necessary deconstruction of an ideal-type

Pascal Fabre, Dominique Bessire et Frédérique Letort

\section{CpenEdition}

\section{Journals}

Édition électronique

URL : http://journals.openedition.org/pmp/7037

ISSN : 2119-4831

Éditeur

Institut de Management Public (IDPM)

\section{Édition imprimée}

Date de publication : 15 avril 2014

Pagination : 193-213

ISBN : 978-2-7430-1997-6

ISSN : 0758-1726

\section{Référence électronique}

Pascal Fabre, Dominique Bessire et Frédérique Letort, « La comptabilité de gestion dans les villes : la nécessaire déconstruction d'un idéal-type », Politiques et management public [En ligne], Vol 31/2 | 2014, mis en ligne le 29 décembre 2016, consulté le 21 décembre 2020. URL : http:// journals.openedition.org/pmp/7037 


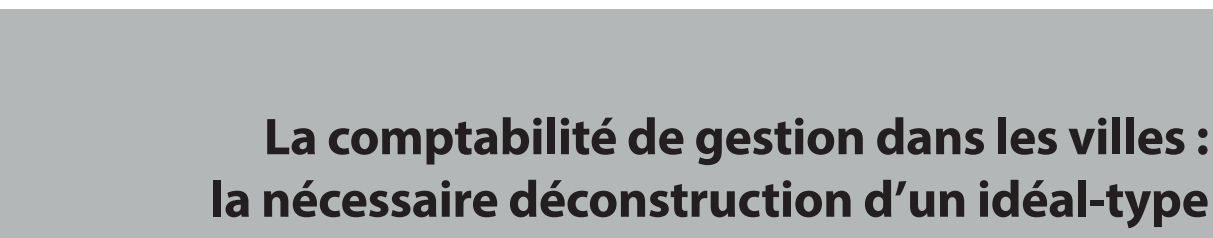

Pascal Fabre ${ }^{a *}$, Dominique Bessire $^{b}$ et Frédérique Letort ${ }^{b}$

aUniversité de Franche Comte, IAE-GREGO

' Université d'Orléans, IAE-VALLOREM

\section{Résumé}

Issu d'une démarche de recherche essentiellement inductive, cet article met en évidence, en croisant la revue de la littérature et l'expérience personnelle des auteurs, l'existence implicite d'un idéal-type pour la mise en place de comptabilités de gestion. L'inadaptation de cet idéal-type aux contraintes spécifiques des villes, permet de comprendre les difficultés rencontrées dans l'implantation des comptabilités analytiques et propose un certain nombre de solutions alternatives.

(c) 2014 IDMP/Lavoisier SAS. Tous droits réservés

Mots clés : villes, comptabilité de gestion, idéal-type, contingence structurelle, démarche inductive.

\section{Abstract}

Management accounting in towns and cities: the necessary deconstruction of an ideal-type. Mainly based on an inductive research methodology this article highlights, by combining the literature and the field experience of the authors, an implicit ideal-type. The inability of such an ideal-type to adapt to the specific constraints of the towns explains the difficulties encountered in the implementation of the analytical accounting systems and calls for a number of alternative solutions.

(c) 2014 IDMP/Lavoisier SAS. Tous droits réservés

Keywords: cities, management accounting, ideal-type, structural contingency, inductive methodology.

*Auteur correspondant : pascal.fabre@univ-fcomte.fr

doi :10.3166/pmp.31.193-213 @ 2014 IDMP/Lavoisier SAS. Tous droits réservés 


\section{Introduction}

Dans un contexte marqué par le resserrement des contraintes financières et la montée des besoins et sous l'influence croissante de ce qu'il est convenu d'appeler le New Public Management (Hood, 1991, 1995), l'on pourrait s'attendre à une diffusion très large des systèmes de comptabilité de gestion au sein des collectivités territoriales. Or, paradoxalement, après une période de bouillonnement à la fin des années soixante-dix et dans les années quatre-vingt qui a vu fleurir les initiatives dans de nombreuses villes, le mouvement a eu tendance à se stabiliser, voire à régresser (Beaulier et Salery, 2006).

Notre article a pour objectif d'éclairer ce paradoxe et d'en tirer un certain nombre d'enseignements qui puissent être utiles aussi bien aux responsables territoriaux qu'aux enseignants et/ou chercheurs. Il s'inscrit dans le courant de la contingence structurelle (Lawrence et Lorsch, 1974) appliqué aux outils de contrôle de gestion et plus particulièrement la comptabilité de gestion. Il résulte également d'une démarche essentiellement inductive et résume les conclusions auxquelles nous avons abouti à la suite de la mise en place, dans le cadre de deux chantiers de recherche-action, d'un système de calcul des coûts dans deux villes de plus de 20000 habitants. En croisant les enseignements de cette démarche de recherche-action avec les constatations d'autres universitaires et praticiens (Gibert, 1995, 2007 ; Demeestère, 2000, 2007), nous avons mis en évidence l'existence implicite d'un «idéal-type » de la comptabilité analytique présent dans la plupart des ouvrages pédagogiques consacrés à la discipline, mais également dans l'esprit des différents intervenants (élus, cadres territoriaux, universitaires, consultants). Cet idéal-type de la comptabilité analytique renvoie à un outil à usage essentiellement interne, aboutissant à des coûts complets, calculés mensuellement, incluant les frais de structure, avec une couverture totale des activités de la collectivité.

La référence à cet idéal-type implicite a deux conséquences perverses. En premier lieu, ce dernier s'avère difficilement conciliable avec les contraintes opérationnelles, financières et politiques auxquelles sont soumis les responsables territoriaux et devient paradoxalement, ainsi pensé, un obstacle au calcul des coûts. En second lieu, perçu comme « la solution normale », il empêche l'émergence de solutions alternatives, mieux adaptées aux contraintes précitées, mais souvent considérées par ceux-là même qui les utilisent comme n'étant pas une « véritable» comptabilité de gestion.

Les développements qui suivent, tentent de décrire la réalité (ainsi que la chronologie) de la démarche de recherche suivie par les auteurs, celle-ci étant de nature quelque peu atypique. En effet nous allons exposer ici dans une première section l'émergence d'une hypothèse comportementale (la focalisation sur un idéal-type) sur la base d'observations de terrain faites sur plus d'un quart de siècle par des professionnels et des enseignants-chercheurs (dont les auteurs) puis nous examinerons dans une deuxième section en quoi cette hypothèse nous a permis de mieux comprendre les difficultés d'implantation de la comptabilité de gestion et sur cette même base et dans le cadre d'une démarche prescriptive nous formulerons dans une troisième section des propositions palliatives à même de faciliter la mise en œuvre d'un tel type d'outil. 


\section{La comptabilité de gestion dans les collectivités territoriales : la référence implicite à un idéal-type}

Pourquoi l'implantation de systèmes de comptabilité de gestion rencontre-t-elle de grandes difficultés dans les collectivités territoriales? Telle est au fond la question à laquelle cet article cherche à répondre. Après une revue de littérature sur le sujet, nous exposerons notre démarche de recherche, très largement inductive et alimentée par deux chantiers de recherche-action ainsi que le premier résultat auquel nous sommes parvenus : la référence implicite à un idéal-type dans toute démarche de mise en place de comptabilité de gestion dans les collectivités territoriales.

\subsection{La transposition problématique d'un outil classique}

L'analyse de la littérature met en évidence des difficultés de déploiement de la comptabilité de gestion dans les collectivités territoriales, sans qu'un consensus se dégage sur les raisons de ces difficultés.

\section{Des difficultés d'implantation et de pérennisation}

L'apparition de la comptabilité de gestion est relativement récente en collectivité. La première expérience recensée dans une ville est celle d'Orléans en 1979. Le contexte y semble alors particulièrement favorable : un maire impliqué dans la gestion et l'aide d'une équipe universitaire spécialiste dans ce domaine. L'objectif est de mettre en place une architecture globale de calcul des coûts afin de planifier les investissements à long terme, de prévoir leur maintenance et leur renouvellement. Ces débuts s'avèrent peu convaincants : le système mis en place est lourd en fonctionnement et en maintenance informatique et il sera définitivement abandonné en 1989 avec le changement de l'équipe municipale (Meyssonnier, 1993).

À partir des années quatre-vingt, trois éléments vont favoriser la diffusion de la comptabilité de gestion dans les villes : d'abord les lois de décentralisation ${ }^{1}$ qui valident le principe de libre administration des collectivités territoriales, ensuite le resserrement des contraintes financières (raréfaction des ressources et montée de besoins), enfin l'irruption dans les collectivités d'une nouvelle génération de cadres territoriaux issus des formations en gestion publiques des universités et des grandes écoles où ils ont intégré de nouvelles pratiques managériales le plus souvent empruntées au secteur privé (Camel, 1987). Pour ces jeunes managers qu'ils soient directeurs généraux des services, directeurs financiers ou contrôleurs de gestion, la comptabilité de gestion semble un outil pertinent à même d'éclairer les dirigeants territoriaux dans leurs prises de décisions.

Les années quatre-vingt-dix sont le moment des premiers retours d'expérience et ceuxci sont plutôt négatifs, un intéressant exemple type étant la ville de Suresnes et ses 2180 centres de coût (Feitz, 1990). Encore mieux documenté est le cas de la Roche-sur-Yon (Bargain, 2012) qui en 1989 mandate ainsi un cabinet de conseil pour mettre en place une comptabilité analytique dans le but de connaître le coût des prestations et les coûts globaux par centre de responsabilité, par service et par direction. Calqué sur celui d'un grand groupe industriel pour lequel un des membres du cabinet de conseil avait travaillé, le système mis

\footnotetext{
${ }^{1}$ Loi du 2 mars 1982 et loi du 7 janvier 1983 dites lois Deferre.
} 
en place, techniquement ambitieux et très lourd au niveau maintenance est abandonné dès 1992. Meyssonnier (1993) constate à cette époque que l'utilité perçue des coûts semble très variable selon les services. Les systèmes qui ont été mis en place sont fondés sur le calcul des coûts complets selon la méthode des sections homogènes ; ils semblent avoir du mal à se pérenniser. Les coûts directs mis en place en fonction des besoins semblent souvent perçus par les fonctionnaires territoriaux comme une solution présentant un meilleur rapport coût/ avantage (Roussarie, 1994). Au final, les systèmes globaux en coûts complets apparaissent donc difficiles à mettre en place dans les villes, les mairies telle Angers ayant réussi avec succès à implanter un tel système (Meyssonnier, 1993) et surtout à le faire perdurer (Bargain, 2011) semblant fort peu nombreuses.

\section{Des débats contradictoires}

Dès le début des années quatre-vingt-dix, des chercheurs analysent ces expériences. Meyssonnier (1993) remarque ainsi une utilisation importante de calculs de coûts ad hoc par les fonctionnaires territoriaux, mais soutient l'intérêt d'un système global en coûts complets se bonifiant avec le temps. Il admet cependant (1993, p. 144) qu' "une comptabilité analytique rénovée en coûts complets peut être très utile aux communes mais [que] son coût est assez important ». Mais pour Gibert (1995), "les coûts d'implantation et de fonctionnement ne posent problème que si l'utilité d'un tel système n'est pas avérée. Or pour lui les systèmes globaux de comptabilité en coût complet sont trop souvent implantés sans une identification correcte des besoins en informations des décideurs territoriaux, ce qui explique l'échec relatif de cet outil en collectivité ».

Roussarie (1994) de son côté pense qu'il faut réserver les coûts complets aux biens à caractère marchand et à bénéficiaire identifiable. C'est également l'avis de Rey (1994) qui rejette les systèmes globaux qualifiés « d'approche par le haut » et cumulant les handicaps :

- succès opérationnels mitigés malgré la « faveur des experts-comptables »;

- non-pertinence de la répartition « fine » des frais généraux;

- méthode longue, onéreuse, difficile à pérenniser, inexplicable aux opérationnels.

Il préconise une « approche par le bas » privilégiant les coûts directs, méthode suffisante du fait du caractère particulier des collectivités (absence de concurrence) et permettant l'élimination des charges non maîtrisables.

Cette première revue de la littérature fait apparaître deux types distincts de débats :

- l'un technique, sur l'intérêt respectif de systèmes globaux en coûts complets par la méthode des sections homogènes ou de calculs de coûts ad hoc, débat qui pose notamment les questions de périmètre de calcul des coûts, de type de coûts (complet, direct, spécifique...), de périodicité de leur calcul (choix entre des calculs ponctuels et un système permanent);

- l'un plus «politique », sur l'utilité réelle d'un tel outil.

Si la comptabilité de gestion semble poser des problèmes tant dans sa mise en place que dans son emploi, elle n'en reste pas moins d'après Gibert (1995) un outil emblématique car marqueur de changement de par sa lourdeur même qui la rend plus visible ainsi que par l'image de rigueur qu'elle véhicule. Elle paraît ainsi « exercer une sorte de fascinationrépulsion chez les responsables territoriaux. Répulsion quand elle leur paraît représenter le type même «d'usine à gaz » dont il faut éviter de se doter. Fascination quand, malgré cela, on voit se développer les systèmes de comptabilité analytique ou que l'on voit ceux qui 
s'en sont dotés sans grand profit faire plus qu'hésiter à les abandonner. » (Gibert, 1995, p. 213). Comme nous allons être amenés à le constater de par nous-mêmes, cette analyse va s'avérer, dix ans plus tard, toujours pertinente.

\subsection{Des situations concrètes imposant des solutions « non conventionnelles »}

Une double confrontation des auteurs avec des « situations limites » lors de deux « recherches action » mais également avec les interrogations des experts du domaine lors d'un séminaire de réflexion, aboutit à la mise en évidence d'un idéal-type relatif au déploiement de la comptabilité de gestion.

\section{La confrontation avec le terrain : des postulats inapplicables}

C'est instruit de ces difficultés d'implantation et de pérennisation, à la fois par la littérature, mais aussi par notre expérience (l'un d'entre nous en particulier intervenant dans le domaine des systèmes de contrôle de gestion en collectivités depuis une vingtaine d'années, notamment au travers d'enseignements, de suivi de stagiaires et d'apprentis), que nous nous engageons en 2004, non sans de fortes appréhensions, dans un chantier de recherche intervention portant sur le calcul des coûts dans une commune de taille moyenne (environ 20000 habitants). En effet la ville de A souhaite mettre en place un système de comptabilité de gestion et sollicite le concours d'universitaires.

Une première difficulté tient à l'objectif même du contrat de recherche. Les discussions avec l'équipe municipale font rapidement apparaître que celle-ci a une priorité : être capable de mettre en avant, auprès de la population, l'effort engagé par la collectivité pour les services rendus. En effet, les bénéficiaires des services municipaux (cantine scolaire, école de musique, piscine...) ont tendance à sous-estimer la valeur du service rendu et considèrent souvent le prix payé comme excessif; de son côté, l'équipe municipale et les opérationnels pressentent, mais sans pouvoir étayer leur ressenti par des chiffres, que le prix payé est significativement inférieur au coût de revient. Il faut donc pouvoir disposer de « coûts de revient complets » permettant à la population de mesurer l'équivalent tarifaire d'une prestation semblable délivrée par le secteur privé. Cette utilisation «politique » du calcul des coûts n'est guère évoquée par les articles de référence sur l'analyse des coûts (Gibert, 1995 ; Demeestère 2007) malgré sa relative fréquence (Fabre, 2005) et ses particularités. En effet, un haut degré de précision n'est pas requis puisqu'il s'agit d'aboutir à des « coûts estimés » avec un objectif de dialogue avec les usagers. Reste qu'un tel objectif, plutôt ambitieux, implique la détermination de coûts complets concernant la grande majorité de services de la collectivité. On se retrouve donc face à une de ces « implantations à risques » bien connues des auteurs mais aussi des cadres territoriaux de la ville de A.

Une deuxième difficulté apparaît alors, précisément liée à cette connaissance commune des risques encourus. En effet le directeur général des services nous demande expressément « d'éviter de monter une usine à gaz », illustration saisissante du couple fascination/répulsion décrit par Gibert en 1995. Ceci revient de facto à nous demander de modéliser une organisation complexe de façon simple, ce qui induit évidemment une contrainte supplémentaire.

Une troisième difficulté tient aux moyens alloués au projet, financiers mais surtout humains, qui sont très faibles. En particulier les ressources en personnel qualifié sont insuffisantes pour effectuer le travail en interne, les services comptables étant monopolisés par 
les tâches courantes liées aux comptes administratifs et à la préparation budgétaire. Au-delà, cette faiblesse des moyens pose le problème (classique en collectivité) de la pérennité du système de calcul des coûts une fois sa mise en place effectuée.

La prise en compte de ces impératifs ainsi que les contraintes précédemment évoquées vont nous conduire à prendre un certain nombre d'options n'entrant pas dans le cadre de la démarche classique de mise en place des sections homogènes (Fabre et Bessire, 2008) :

- nous avons ainsi renoncé à mettre en place un système permanent de saisie des charges avec détermination de coûts annuels ou trimestriels trop lourd et opté plutôt pour un calcul de coûts unique avec possibilité de réactualisation pluriannuelle (3 à 5 ans). Il s'agissait en effet de produire des coûts estimatifs et l'importance des charges fixes rendait leur évolution relativement lente. Ceci a évité de mobiliser des moyens financiers et humains de façon permanente;

- nous avons par ailleurs défini une architecture des coûts simplifiée, en définissant un nombre limité de centres d'analyse;

- nous avons enfin porté plutôt l'effort sur la fiabilisation des coûts directs en optant pour des clés de répartition simplifiées.

Nous remettrons notre rapport de fin de mission le 10 octobre 2005 et la mise en place opérationnelle du système de calcul des coûts s'achèvera en juin 2006.

Au printemps 2009, l'adjoint aux finances de la ville de A, qui entre-temps est devenu adjoint aux finances de la ville de $\mathrm{B}$ (de taille similaire à la ville de $\mathrm{A}$ ), nous sollicite pour mener le même projet dans la ville qu'il a rejointe. Le système de calcul des coûts sera opérationnel dès juin 2011. La conduite de cette nouvelle recherche action sera l'occasion de faire une synthèse des enseignements recueillis et de confirmer la pertinence des choix effectués, notamment les libertés prises avec le processus « classique » de détermination des coûts.

\section{La confrontation avec les experts : des analyses fragmentaires mais éclairantes}

Au cours de ces deux chantiers de recherche-intervention, nous avons rassemblé un riche matériau empirique. Encore fallait-il lui donner du sens. Tout au long de ce travail, nous avons poursuivi la revue de littérature et les échanges avec d'autres chercheurs ou professionnels impliqués dans ce genre de projet. Un séminaire organisé par Gibert (2007) portant sur la comptabilité analytique dans les collectivités locales et réunissant de nombreux spécialistes de la question va donner un éclairage nouveau à notre travail.

Lors des exposés, l'utilisation de calculs de coûts ponctuels en lieu et place d'un système permanent est notamment plusieurs fois évoquée mais plutôt comme solution alternative « faute de mieux » et cette solution est carrément rejetée par certains intervenants reprenant le point de vue déjà exprimé antérieurement par Meyssonnier (1993). L'utilisation « politique » de l'outil analytique comme instrument d'argumentation et de valorisation de l'action publique n'est également que très peu abordée, l'accent étant mis sur l'utilisation opérationnelle (contrôle de l'efficience) et stratégique de l'outil (aide à la décision). Or notre expérience des collectivités territoriales, au travers de nos deux chantiers de recherche action, mais aussi d'encadrements de stagiaires et d'apprentis durant une vingtaine d'années ainsi que d'autres travaux de recherche (Fabre, 2005) nous a montré que cette utilisation est fréquente en collectivité.

À la lumière de ces échanges, il semble donc qu'il y ait une façon «classique », « normale », « orthodoxe », de calculer les coûts, en mettant en œuvre un système permanent, 
avec des objectifs opérationnels et/ou stratégiques, l'outil étant censé répondre à l'ensemble des informations portant sur les coûts. Le retour à la littérature, aux ouvrages fondateurs de Bouinot (1977), de Carles et de De Kerviler sous l'égide du Ministère des collectivités locales (1988) et aux travaux plus récents de Bargain $(2011,2012)$ va faire naître une intuition : ce qui lie ces dispositifs pratiques, ces ouvrages et travaux, c'est la référence implicite à un « idéal-type » de la comptabilité de gestion.

\subsection{L'émergence d'un modèle explicatif cohérent}

La notion d'idéal-type ou de type idéal trouve son origine chez Max Weber (1965). Selon le sociologue, "l'idéal-type est un tableau de pensée, il n'est pas la réalité [...]. Il n'a d'autre signification que d'un concept limite purement idéal, auquel on mesure la réalité pour clarifier le contenu empirique de certains de ses éléments importants, et avec lequel on la compare ». Coenen-Huther (2003), le définit comme le résultat de la conceptualisation d'un objet, dans une version stylisée de la réalité.

S'agissant de la comptabilité de gestion dans les collectivités locales, nous allons montrer que cet idéal-type s'inscrit dans l'histoire des Sciences de Gestion et nous nous efforcerons ensuite d'en décrire les principales caractéristiques.

La construction historique d'un idéal-type : la référence à l'entreprise industrielle

Il semble que les racines de cet idéal-type plongent dans les travaux menés dès la Révolution industrielle. À cette époque, le calcul des coûts, s'est développé au sein des entreprises dans le but selon Nikitin (1992), d'aider à la fixation des prix de vente. En 1927, le lieutenant-colonel Rimailho, mandaté par la CEGOS, présente une méthode de calcul de coûts complets. Cette méthode sera ensuite proposée sous le nom de « méthode des sections homogènes » dans le premier Plan comptable en 1947 (puis dans le PCG 1982 sous le nom de « centres d'analyse »). Trouvant dans le PCG un support idéal à sa diffusion auprès des professionnels de la comptabilité, la méthode s'impose donc progressivement en France dans les entreprises, dans l'enseignement et dans les cabinets d'expertise-comptable. La référence est alors l'entreprise industrielle caractérisée le plus souvent par une activité mono-produit, des charges majoritairement directes (main-d'œuvre directe) et l'importance des consommations matières.

Dans les villes, la comptabilité de gestion apparaît beaucoup plus tardivement. Le premier ouvrage notable sur la comptabilité analytique dans les communes est celui de Bouinot (1977), un universitaire orléanais spécialiste de la gestion des collectivités locales, celui-là même qui a aidé la ville d'Orléans à mettre en place à la fin des années soixante-dix son système de comptabilité de gestion. L'étude de la bibliographie montre l'importance des ouvrages de comptabilité de gestion anglo-saxonne axés essentiellement sur les process des entreprises industrielles.

En 1988, un guide professionnel, émanant du Ministère des collectivités locales et publié à la Documentation française à destination des communes, est entièrement consacré à la comptabilité analytique. Les rédacteurs, Carles et De Kerviler, sont deux experts-comptables qui s'appuient sur les expériences en la matière de 13 communes $^{2}$. Même si les

\footnotetext{
${ }^{2}$ Châtillon, Chartres, Issy-les-Moulineaux, La Roche-sur-Yon, Montbéliard, Nancy, Rezé, Orléans, SaintOuen-L'aumône, Sucy-en-Brie, Turennes, Troyes, Tourcoing.
} 
auteurs reconnaissent l'existence de caractéristiques propres aux collectivités, la méthode préconisée, uniforme, est celle de la méthode des coûts complets par les centres d'analyse (PCG 1982); autrement dit un système global au niveau de l'ensemble des services (ou divisions) de la commune. En l'absence à cette époque d'autres ouvrages similaires ${ }^{3}$ il n'est pas interdit de penser que les écrits de ces auteurs ont eu une influence déterminante sur la conception de systèmes de comptabilité de gestion dans les communes. De plus, le système d'enseignement supérieur (dont on a vu précédemment qu'il avait formé de nombreux jeunes cadres territoriaux) a également probablement joué un rôle normatif de par la très grande place donnée à la comptabilité analytique en coût complet. Ainsi un examen approfondi des annales des examens comptables (DECS) pour les années 70/80 montre que la référence reste à l'époque la méthode des sections homogènes (avec des applications essentiellement en milieu industriel). Enfin la problématique de la contingence des modèles de comptabilité analytique et de leur adaptation aux particularités des organisations reste à l'époque un champ peu documenté et peu enseigné. On peut renvoyer à ce sujet à l'analyse de Gibert et Thoenig (1992) qui soulignent l'importance de la formation initiale comme vecteur de diffusion des outils de gestion pour les cadres supérieurs du secteur public.

\section{Sa caractérisation}

L'ensemble des débats précédemment évoqués et ce bref parcours historique permettent d'établir un tableau récapitulatif (tableau 1) recensant les principales caractéristiques de cet idéal-type et mettant en parallèle les choix alternatifs possibles.

Tableau 1 : Principales caractéristiques de l'idéal-type

\begin{tabular}{|l|l|}
\hline \multicolumn{1}{|c|}{ L'idéal-type } & \multicolumn{1}{c|}{ Et ses alternatives } \\
\hline $\begin{array}{l}\text { Un système permanent avec des calculs de coûts } \\
\text { réguliers }\end{array}$ & Des calculs de coûts ponctuels \\
\hline $\begin{array}{l}\text { Une couverture exhaustive et homogène de la } \\
\text { collectivité }\end{array}$ & $\begin{array}{l}\text { Une approche différenciée et sélective suivant } \\
\text { les besoins des services }\end{array}$ \\
\hline Des coûts complets intégrants les frais de structure & $\begin{array}{l}\text { Des coûts directs à l'équipement, au service, } \\
\text { à l'activité }\end{array}$ \\
\hline $\begin{array}{l}\text { Une utilisation opérationnelle et/ou stratégique } \\
\text { des calculs de coûts }\end{array}$ & Une utilisation politique des calculs de coûts \\
\hline
\end{tabular}

Source : les auteurs

La lecture de ce tableau met en évidence l'ensemble des postulats sous-jacents à l'idéaltype. Celui-ci correspond à une mise en œuvre considérée comme «normale » de l'outil, une situation optimale vers laquelle il faut tendre (Meyssonnier, 1993) même si les contraintes opérationnelles et politiques imposent une mise en place progressive (Ministère des collectivités locales, 1988). Comme le souligne Meyssonnier (p. 223), « une comptabilité analytique globale : c'est le calcul de coûts complets (et pas de coûts simplement partiels directs) dans toute l'organisation (et pas seulement cantonnés au centre technique municipal) ». À l'extrême, certains avancent même l'idée d'une conception «dogmatique » de la mise en œuvre de la

${ }^{3}$ D'autres ouvrages (Dupuis; Allègre et Mouterde; Rey) sortent la même année mais ils sont consacrés au contrôle de gestion et donc moins spécialisés. 
comptabilité analytique en collectivités territoriales (Bargain, 2011). Or à la lumière des différents débats évoqués, complétée par notre propre expérience de terrain, l'ensemble de ces postulats semble contestable ou pour le moins parfois difficilement applicable. En témoigne l'option fréquemment prise par les opérationnels des choix alternatifs précédemment exposés.

\section{L'idéal-type de la comptabilité de gestion : une méconnaissance de la triple rationalité à l'œuvre dans les collectivités territoriales}

Reste à comprendre en quoi la comptabilité de gestion, telle qu'elle est pensée dans son idéal-type, pose des difficultés. De nombreux facteurs ont été évoqués pour expliquer l'échec relatif de l'implantation de l'outil analytique dans les villes. Gibert (2007) avance, par exemple, l'irréalisme des objectifs, des moyens financiers limités, le manque d'expertise au niveau local. Au-delà de ces explications partielles, c'est à notre avis dans l'application simultanée de l'ensemble des postulats composant l'idéal-type qu'il faut chercher la source majeure des échecs enregistrés. Cet idéal-type fait en effet fi des trois types de rationalité à l'œuvre dans les collectivités territoriales : opérationnelle, économique et politique (Cellier et Chatelain-Ponroy, 2005).

Tableau 2 : Les trois grands types de rationalité des organisations publiques

\begin{tabular}{|c|c|c|c|}
\hline Rationalité & Politique & Économique & Opérationnelle \\
\hline Définition & $\begin{array}{c}\text { Exprimée par les missions } \\
\text { de services publics. } \\
\text { Volonté des élus de } \\
\text { satisfaire leurs administrés. }\end{array}$ & $\begin{array}{c}\text { Nécessité de réguler, de } \\
\text { contrôler les dépenses } \\
\text { publiques. }\end{array}$ & $\begin{array}{c}\text { Portée par les acteurs } \\
\text { opérationnels, prestataires } \\
\text { de services. }\end{array}$ \\
\hline Objectif & Efficacité & Efficience & Qualité \\
\hline
\end{tabular}

Source : adapté de Cellier et Chatelain-Ponroy (2005).

\subsection{Une inadaptation aux contraintes opérationnelles}

Sur le plan opérationnel, l'idéal-type s'avère assez largement inadapté. En particulier du fait de la faible utilité d'un calcul de coût pour de nombreuses activités, une comptabilité de gestion assurant une couverture exhaustive de la collectivité apparaît peu pertinente. De plus compte tenu des caractéristiques des collectivités, le calcul d'un coût complet intégrant les charges de structures, n’a en général pas une grande utilité.

\section{Des activités trop diverses}

Pour Mintzberg (1982), les mairies sont amenées à gérer des structures de type très varié (de forme adhocratique pour les services d'aménagement du territoire, relevant de la bureaucratie professionnelle pour la police municipale ou avec une structure de bureaucratie mécaniste pour le centre technique municipal, pour ne citer que quelques exemples). Leur organisation interne par « directions métiers » les rapproche souvent des structures divisionnelles (avec toutefois une tendance permanente à dériver vers une bureaucratie de type mécaniste). C'est aussi la conclusion de Meyssonnier (1993) qui assimile le système communal à un conglomérat mais un conglomérat qui ne bénéficie pas de la logique intégrative que procure la recherche du profit. 
Il est rejoint par Gibert (1995) pour qui cette analyse n'est pas dépourvue de pertinence du fait de la variété et souvent de l'absence de liens entre les différentes activités communales. Une telle analyse plaide pour des systèmes de contrôle différenciés selon les secteurs d'activité en fonction de leurs caractéristiques et des besoins d'informations des responsables de service. Or comme nous allons le voir, l'intérêt d'un calcul des coûts s'avère très limité pour beaucoup d'activités. Ceci remet en cause l'utilité de mettre en place un système global de comptabilité analytique sauf dans l'optique de communes où le contrôle de gestion est centré sur cet outil avec une priorité à la recherche de l'efficience des services. Or cette configuration s'avère, selon l'analyse de Meyssonnier (1993), plutôt marginale, les préoccupations des élus étant autres, en particulier la recherche de l'efficacité mieux assurée par l'outil « tableaux de bord ».

\section{Une utilité limitée}

Concernant la nécessité d'un coût complet intégrant les charges de structure et en reprenant l'analyse de Demeestère (2000), il convient de savoir si cette caractéristique de l'idéal-type est bien adaptée à l'usage que l'on entend faire de la comptabilité de gestion.

Concernant la tarification et comme on l'a signalé précédemment, la détermination d'un coût complet peut être particulièrement pertinente pour des activités quasi marchandes où l'intégralité des charges doit être couverte par l'usager (eau, ordures ménagères). Reste qu'en collectivité, on est confronté à des activités généralement non marchandes (pas de prix réel) et dont les bénéficiaires sont parfois non identifiables (pas de facturation possible). L'essentiel du financement est assuré par l'impôt, contrepartie du bouquet de services et d'activités offerts par la mairie, le rapport coût/avantage de l'ensemble étant évalué par l'électeur/contribuable au moment des élections. Dans cette configuration et en l'absence de concurrence la détermination d'un coût complet par activité pour aider à la tarification ne s'impose généralement pas.

Concernant le choix public/privé et en admettant même qu'une telle comparaison soit possible, l'utilisation du coût complet peut s'avérer dangereuse. En effet à quoi servent les coûts s'il n'y a pas fongibilité des moyens, rappelle Gibert (2007). Bien évidemment, les collectivités n'ont qu'une latitude restreinte vis-à-vis de leur personnel du fait des protections statutaires. Et pour ce qui est des équipements, ceux-ci sont souvent dédiés et difficilement réaffectables.

Concernant le contrôle de l'efficience des services, l'accent doit être mis sur les charges maîtrisables par les responsables territoriaux, ce qui n'est pas le cas des charges de structures qui sont des charges subies. Dans ces circonstances, l'utilisation d'un coût complet difficilement explicable peut décrédibiliser l'ensemble de la démarche de contrôle des coûts. C'est l'exemple caricatural du stylo du cabinet du maire qui se retrouve pour 4/10000 dans le prix de revient d'une entrée à la piscine municipale (Rey, 1994).

On le voit, dans de nombreuses configurations (mais pas toutes), le calcul d'un coût complet exigé par l'idéal-type est un investissement peu souhaitable.

\subsection{Des contraintes financières ignorées}

L'exigence d'un système exhaustif, caractéristique de l'idéal-type, s'avère excessivement coûteuse à l'implantation dans le contexte d'une organisation à activités multiples. Elle augmente également de façon notable les moyens à engager pour la mise en œuvre du système, moyens à engager chaque année du fait de la nécessité imposée d'un système permanent. 


\section{Une démarche trop coûteuse à l'implantation}

La mise en place d'une comptabilité analytique couvrant l'intégralité des services dans une ville, même de taille moyenne, est un processus long et donc fort coûteux. Allègre et Mouterde (1991) comptent, pour une comptabilité analytique sur l'ensemble d'une collectivité, un an d'étude auquel il faut ajouter deux ans de validation opérationnelle pour roder et fiabiliser le système. Le coût lié à la mise en place peut donc s'avérer extrêmement élevé d'autant que la multiplicité des activités réalisées par les communes ne facilite pas la tâche.

Il faut, soit disposer en interne de personnel qualifié et disponible sur longue période (mais les personnels comptables de haut niveau sont difficiles à trouver en collectivité), soit faire appel à un prestataire extérieur dont les tarifs sont souvent hors de portée des possibilités financières d'une commune. En effet la structure de coût des cabinets conseils du secteur privé, n'est pas réellement adaptée à des missions de cette nature. Ceux-ci utilisent généralement une main-d'œuvre très qualifiée imposant un taux de facturation élevé et donc des devis dépassant les capacités de la collectivité pour une mission aussi fortement consommatrice en temps de main-d'œuvre (en particulier lors de la phase de collecte d'information en vue de la répartition primaire).

\section{Un rapport coût/avantage médiocre à l'exploitation}

Gibert (1995, p. 116) estime que le rapport coût/avantage est défavorable pour les communes en cas de non-utilisation des résultats produits, mais que sinon le coût du système ne doit pas être un frein à sa mise en place : "C'est le rapport utilité/coût du système d'information mis en place qui est, le cas échéant, critiquable, non le coût lui-même, nécessairement élevé, qui constitue la contrepartie d'une certaine fiabilité de l'information ». On retrouve ce scénario à Orléans avec, d'après Meyssonnier (1993), un système lourd en fonctionnement et en maintenance pour des résultats peu exploités (autrement dit un rapport coût/avantage médiocre), avec à terme un abandon du dispositif.

Mais justement un tel rapport coût/avantage ne semble généralement pas apparaître comme suffisant aux acteurs territoriaux. C'est ainsi que la Cour des Comptes (2009) peut affirmer que concernant les collectivités locales la tenue d'une comptabilité analytique complète (concernant l'ensemble de l'organisation) apparaissait à beaucoup comme lourde, coûteuse et contraignante à bâtir sans permettre de rendre compte de façon satisfaisante de la performance de la collectivité. Il faut dire que dans nombre de cas, la priorité des politiques va sans doute plus à la satisfaction des électeurs qu'à l'efficience des services. Or comme nous allons le voir, ce sont eux qui vont décider de la mise en place et du maintien d'un tel outil.

\subsection{Une logique politique non prise en compte}

L'exigence d'exhaustivité de l'idéal-type va faire de la mise en place d'une comptabilité analytique, un projet lourd et coûteux nécessitant un soutien fort des élus. Or leurs préoccupations relevant surtout de l'ordre du politique sont mal prises en compte par l'idéal-type, l'utilisation normale de l'outil relevant des champs opérationnels et stratégiques.

\section{La nécessité d'un soutien fort des politiques}

L'idéal-type impose une mise en place de la comptabilité de gestion sur l'ensemble des activités de la collectivité. C'est «l'approche par le haut » évoquée par Rey (1994). Ceci fait de cette mise en place un projet lourd qui va nécessiter un soutien fort du « top management» 
de la collectivité, en l'occurrence le maire (et dans une moindre mesure son directeur général des services). Ce sont également ces acteurs qui peuvent décider du maintien ou de l'abandon de cet outil. À cet égard les directeurs financiers et les éventuels contrôleurs de gestion ne jouent qu'un rôle de prescripteurs. Il en est tout autrement dans le cas « d'une approche par le bas » (Rey, 1994). Dans ce cadre, ce sont les responsables opérationnels qui jouent un rôle moteur. À ce niveau une sélection « darwinienne » va s'opérer et seuls les responsables des services pour lesquels l'information «coût» est pertinente se lanceront dans un tel investissement. Cette pertinence dépend largement des particularités opérationnelles du service : présence d'une tarification (école de musique), d'importants coûts variables (restauration), de financeurs extérieurs comme la CAF (crèches), de partenaires à soutenir (associations sportives et culturelles, cf. Fabre, 2005). La configuration est tout à fait différente pour un élu dont les préoccupations et les contraintes sont d'une autre nature, beaucoup moins opérationnelle et beaucoup plus politique. Or il n'est pas sûr que les politiques soient motivés par la comptabilité analytique vu le faible avantage qu'ils peuvent en retirer personnellement eu égard à la mobilisation de ressources nécessaire sans compter les risques inhérents à l'outil.

\section{Une orientation inadaptée à leurs préoccupations et porteuse de risques}

À cet égard Chapet (2007) souligne que les outils de gestion ne pénètrent dans l'action publique qu'à condition de servir le politique. Dans ce cadre, la comptabilité analytique utilisée de façon traditionnelle (pour l'optimisation de la gestion interne de la collectivité) n'a d'intérêt pour les élus que dans sa phase de mise en place (permettant ainsi de mettre en avant une image de gestionnaire rigoureux). Au-delà l'utilisation pérenne de l'outil s'avère moins intéressante pour l'élu. Chapet souligne de plus la tendance des équipes de direction dans ces mêmes collectivités à tenir à distance tout outil risquant de fragiliser leur pouvoir. Or c'est bien le cas de la comptabilité de gestion qui risque de mettre en lumière une allocation parfois peu optimale des ressources municipales. Comme le soulignent Beaulier et Salery (2006), la quantification des moyens engagés met en lumière des déséquilibres difficiles à justifier, que l'absence de données permettait de gérer plus facilement. Cette grille d'analyse permet sans doute de mieux comprendre le développement apparemment limité des comptabilités de gestion globales ainsi que l'abandon d'un certain nombre d'entre elles après une mise en place à grands frais. Il s'agit maintenant d'examiner au regard des recherches actions menées et de la revue de la littérature effectuée, l'intérêt des solutions alternatives à l'idéal-type ainsi que leurs limites.

\section{Du contournement de l'idéal-type : I'alliance du politique et de l'opérationnel}

Si nos deux projets de mise en place de comptabilité de gestion exposés précédemment ont pu être menés à bien, c'est qu'intuitivement, nous nous étions affranchis des postulats de l'idéal-type. Dans cette dernière section, en intégrant notre expérience et les réflexions d'autres chercheurs et de professionnels, nous posons les jalons qui devraient permettre à l'avenir aux responsables territoriaux de s'engager dans une démarche plus cohérente avec les objectifs impartis et les moyens disponibles.

Le contournement des postulats de l'idéal-type peut s'effectuer selon trois axes, de nature différente. Il peut d'abord être nécessaire, en fonction des circonstances, d'aider à l'appropriation de l'idéal-type par les acteurs en politisant l'outil analytique. Il est également très souvent pertinent 
d'adapter l'idéal-type aux caractéristiques opérationnelles des villes par une approche plus sélective des services à couvrir et une simplification des coûts calculés. Enfin, il peut s'avérer opportun de renoncer à un système permanent de calcul des coûts au bénéfice d'approches alternatives.

\subsection{Favoriser l'appropriation par les élus : une utilisation politique de l'outil}

Un premier axe consiste à intégrer la dimension politique de l'action municipale au niveau de l'outil, d'une part en valorisant l'utilisation politique des informations obtenues, d'autre part en évitant le piège de la transparence.

\section{Un outil politique plutôt qu'un outil de pilotage}

L'utilisation d'outils de gestion, et en particulier la comptabilité analytique pour la valorisation de l'action publique et la négociation avec les autres parties prenantes est très fréquente. Ainsi dans une recherche précédente concernant les communes (Fabre, 2005) nous avions pu constater que près des $2 / 3$ des 250 communes interrogées avaient mis en place des systèmes d'information centralisés relatifs aux ressources allouées aux associations (en termes de subventions, mises à disposition et prestations gratuites) avec pour objectif de faire prendre conscience aux associations de l'effort réel de la commune et de son coût pour elle (un système de chiffrage de ce coût existant dans plus de $50 \%$ des communes interrogées ${ }^{4}$ ). En rendant possible des comparaisons interassociations, il permet aussi aux politiques de mieux gérer les demandes toujours croissantes des partenaires associatifs.

Ce type d'usage est bien évidemment particulièrement intéressant pour les élus dont une des missions est d'arbitrer entre les différentes demandes des parties prenantes. Mais il n'est pas sûr que cette forme d'utilisation politique de l'outil analytique soit très souvent mise en avant par les promoteurs de la comptabilité de gestion qui sont souvent des fonctionnaires territoriaux. Or d'autres usages de l'outil impliquant les politiques peuvent être envisagés à l'exemple des mairies A et B. Dans les deux cas les objectifs se sont avérés assez semblables : mettre en valeur l'action de la municipalité, faire prendre conscience aux usagers de l'effort consenti, négocier au mieux avec les partenaires associatifs et la caisse d'allocations familiales. Si le projet était porté pour A part le directeur général des services et pour B par l'adjoint aux finances, à chaque fois le projet a soulevé l'intérêt des acteurs politiques (le maire, son cabinet et ses adjoints) car utilisable par eux-mêmes dans leurs rapports avec les « usagers électeurs contribuables », mais également avec les autres parties prenantes (CAF, associations...). À notre sens, un tel usage garantit mieux la pérennité d'un outil analytique, le politique trouvant son intérêt à la perpétuation de l'outil.

\section{Des résultats diffusés de manière discrétionnaire plutôt qu'une transparence des coûts}

Il ne faut pas sous-estimer le danger pour un politique d'une information sur les coûts largement diffusée. On le voit régulièrement à l'occasion des innombrables articles de presse, souvent comparatifs, consacrés au prix de l'eau ou au tarif de la cantine scolaire. Il s'agit pourtant d'un des rares services municipaux dont les tarifs doivent couvrir les coûts, ceuxci étant donc connus du public. En effet le contrôle politique, mode classique d'attribution

\footnotetext{
${ }^{4}$ Nous avons encore cette année encadré une stagiaire à qui avait été confié ce type de mission dans une commune de moins de 5000 habitants.
} 
des ressources dans les collectivités locales (Hofstede, 1981) peut engendrer des disparités importantes entre les allocataires. Dès lors, révéler de telles disparités implique de pouvoir les justifier, exercice particulièrement difficile qui risque en cas d'échec d'augmenter la conflictualité au sein de l'organisation. C'est ce que nous avons pu constater dans une partie de notre étude (Fabre, 2005) sur les mairies consacrées aux systèmes de suivi centralisé des moyens alloués aux partenaires associatifs valorisés ou non. Dans les municipalités interrogées, d'après les renseignements recueillis, lorsque l'information existe à ce niveau, il semble qu'il y ait une certaine réticence à l'utiliser de façon systématique et plus encore à rendre cette information publique ${ }^{5}$. Ceci s'explique, car à cette occasion, il est effectivement souvent mis en évidence des disparités importantes dans les moyens alloués, difficiles à justifier par rapport à l'activité déployée. Les informations collectées sont donc généralement utilisées de façon ciblée lors des face-à-face entre élus et responsables associatifs pour valoriser au plus juste l'effort consenti par la municipalité en intégrant les mises à disposition et prestations gratuites et pouvoir ainsi diminuer les exigences des partenaires associatifs, notamment en matière de subvention. Il en est de même pour ce qui est des comptabilités analytiques mises en place dans les deux collectivités $\mathrm{A}$ et $\mathrm{B}$ précédemment évoquées où les informations collectées servent de la même façon à la négociation individuelle avec les associations ou dans les discussions individuelles avec les usagers, particulièrement pour leur faire prendre conscience du coût réel de la prestation souvent confondu avec le tarif de cette même prestation. Les coûts calculés n'ont jamais été rendus publics malgré le projet original du directeur général des services de A d'en faire une partie d'un système d'information à destination des « usagers électeurs contribuables ». De fait, l'usager mécontent doit pouvoir apprécier l'effort consenti, mais il vaut mieux que le contribuable électeur et l'opposition politique l'ignorent. Il s'agit donc, à notre sens, de pouvoir garantir aux politiques un accès régulé à l'information sur les coûts de façon à ce qu'ils puissent en bénéficier sans craindre une exploitation à des fins polémiques (le raisonnement étant identique pour l'évaluation de politiques publiques qui rencontre les mêmes problèmes).

\subsection{Simplifier la mise en œuvre opérationnelle : une approche différenciée par services}

Un autre axe consiste à abandonner l'idée même d'une démarche globale au profit d'une approche différenciée par services rejoignant ainsi l'analyse de Rey (1994).

\section{Des services ciblés plutôt qu'une couverture exhaustive}

On peut se demander a contrario de l'analyse d'Allègre et Mouterde (1991) si la comptabilité analytique n'est pas, du fait de la diversité des activités assurées par les villes, un outil à dimension sectorielle plutôt qu'un outil nécessitant une approche globale au niveau de la collectivité. L'approche par le bas préconisée par Rey (1994) semble plus adaptée au cadre communal. C'est aussi l'avis de la Cour des Comptes (2009) qui constate que du fait du faible rapport coût/avantage d'un système global, les recherches de connaissance des coûts, du moins, celles portées à leur connaissance, sont parfois moins ambitieuses et plus ciblées (autrement dit limitées à certains services).

\footnotetext{
${ }^{5}$ Là encore notre plus récente expérience d'encadrement de stage confirme ce constat.
} 
À notre sens, le calcul des coûts en collectivité est d'ailleurs probablement plus développé que ce qu'avancent certains auteurs. En effet de telles démarches localisées au niveau du service sont beaucoup moins visibles et également moins médiatisées, car plus modestes. Et comme le soulignait Meyssonnier (1993), le résultat des enquêtes doit être pris avec précaution, beaucoup de communes considèrent en effet qu'elles ont une comptabilité analytique même si celle-ci est limitée à un ou deux services. Même dans le cas des villes A et B où la volonté était forte de calculer des coûts sur l'ensemble des équipements, activités et services, avec le plein accord de l'équipe dirigeante, l'analyse est restée très sommaire pour certains services telle la police municipale où l'utilité d'un calcul des coûts n'était pas flagrante. Sur certaines activités, selon les objectifs poursuivis, le calcul des coûts peut n'avoir qu'un intérêt très limité, dès lors pourquoi le calculer?

\section{Des coûts directs plutôt que des coûts complets}

On peut réellement douter de l'intérêt de calculer un coût complet en collectivité, celui-ci ressortant plutôt de l'exception que de la «normalité ». Concernant les charges de structure, les collectivités ont d'ailleurs, à travers leurs relations avec les grands délégataires (en particulier dans le domaine de l'eau) une expérience souvent douloureuse du caractère parfois arbitraire de la répartition de telles charges indirectes. Ce caractère arbitraire est, à notre avis, renforcé du fait du modèle économique sous-jacent des villes. L'objectif d'une ville est, en effet, d'offrir un « bouquet » de services et d'activités très divers à ses membres, l'essentiel de la tarification étant forfaitaire par le biais de l'impôt. À ce titre, on est proche de la logique économique d'un parc d'attractions ou d'un cablo-opérateur, voire d'une banque ou d'un grand hôtel (ou la tarification est moins globale). Dans ces configurations, il n'y a généralement pas répartition des charges de structure. Ainsi dans les villes de A et $\mathrm{B}$, le découpage permet d'examiner, selon la finalité recherchée, soit le coût direct, soit le coût « complet », en insistant auprès des acteurs sur le caractère arbitraire des imputations (il s'agit d'avoir un ordre d'idée plutôt qu'un « vrai » coût).

De plus nous avons opté volontairement pour une simplification maximale de la répartition des charges de structure d'un meilleur rapport coût/avantage alors même que nous avions l'outil pour procéder à une répartition beaucoup plus « fine » de type ABC. Les centres correspondant aux services fonctionnels et à la direction générale ont finalement été limités à une dizaine (le strict nécessaire pour assurer la pertinence de l'analyse sans trop la complexifier) et répartis (après consultation des chefs de service et des élus) au prorata des charges de personnel des centres opérationnels. Il s'agissait ici de pouvoir facilement expliquer la répartition des services fonctionnels à la fois aux fonctionnaires territoriaux et (en cas de besoin) aux usagers. Une complexité accrue aurait été contreproductive eu égard à l'objectif de déterminer un coût représentant une bonne estimation de la consommation de ressources (mais pas plus) et de pouvoir facilement expliquer le mode de calcul aux différentes parties prenantes.

Autrement délicat est le problème des prestations internes (notamment les services techniques) qui peuvent représenter une consommation de ressources importantes. Or il s'agit bien de coûts directs au service, à l'équipement ou à l'activité. Mais ce problème peut être résolu par des études ponctuelles sur les équipements problématiques, sans forcément passer par un système permanent de suivi de la main-d'œuvre parfois politiquement délicat à mettre en œuvre. 


\subsection{Opter pour des calculs ponctuels et des mesures extra-comptables}

Un dernier axe plus radical revient à abandonner toute idée de système permanent de calcul de coûts. Dans de nombreuses configurations, des calculs ponctuels ou même un recours aux indicateurs extra-comptables peuvent remplir les mêmes usages pour un coût global très inférieur. Là aussi l'exemple des mairies A et B s'avère particulièrement éclairant

\section{Des calculs ponctuels plutôt qu'un système permanent}

L'intérêt d'une telle alternative ressort clairement du séminaire de 2007. D'après Gibert la mise en place d'un système permanent relève souvent d'un excès d'ambition (eu égard aux moyens disponibles). À la place, il est tout à fait envisageable de faire des analyses ponctuelles et de vivre sur cela pendant plusieurs années. C'est l'optique que nous avons choisie dans le cadre des mairies A et B en préconisant une réactualisation des coûts pluriannuelle (3 à 5 ans). Ceci permettait de résoudre le problème engendré par l'absence de moyens humains et financiers nécessaires au fonctionnement d'un système permanent. En effet, à notre sens, dans le cadre des mairies $\mathrm{A}$ et $\mathrm{B}$, la nature de la mission (obtenir des coûts « estimés ») et la structure même des coûts faiblement évolutive du fait de l'importance des charges fixes, rendaient peu utile un système permanent de calcul des coûts.

Le recours à des calculs ponctuels peut surprendre du fait de la non-actualisation des données et de la précision a priori inférieure à celle d'un système permanent. Mais justement une telle précision est-elle toujours requise dans le cadre des collectivités ? Si l'on se réfère à Sandretto (1985), de nombreux éléments (présents dans les collectivités) rendent inutiles l'obtention de calculs précis et permettent l'utilisation de valeurs approximées. C'est le cas en particulier de l'absence de concurrence, de l'importance des coûts fixes, de la faible complexité de la majorité des produits et services délivrés et de la non-utilisation des coûts pour fixer les tarifs (à l'exception notable de services à caractère «marchand » comme l'eau et les ordures ménagères). Ainsi une comptabilité de coûts par équipement, lourde et coûteuse, peut sans doute être avantageusement remplacée par des calculs de coûts ponctuels permettant de mieux estimer les frais de fonctionnement des équipements actuels et surtout à venir. Il en est de même pour les services techniques, en mettant en œuvre une refacturation interne de leurs prestations sur la base de taux horaires standards issus de calculs de coûts ponctuels à réactualisation pluriannuelle.

\section{Des mesures physiques plutôt que des mesures comptables}

Vu l'importance des coûts fixes en collectivité et le caractère limité des ajustements possibles, la valorisation du coût d'un équipement ou d'une activité n'est pas forcément un atout L'important est parfois plus de connaître le nombre d'heures d'utilisation de l'équipement et le nombre de personnes utilisatrices par heure. L'idée n'est pas nouvelle et a déjà été développée par Meyssonnier (1993) qui cite l'exemple de l'état civil où un calcul régulier des coûts ne s'impose pas et peut être remplacé par des relevés de fréquentation permettant le calcul de ratios d'efficience. Il est vrai que les indicateurs extra-comptables (en particulier ceux de fréquentation) sont un substitut intéressant de la valeur créée et permettent en les rapprochant des coûts un contrôle plus efficace de l'efficience. À cet égard, les coûts calculés dans les mairies de A et B ont été systématiquement rapprochés d'indicateurs physiques tels que le nombre de bénéficiaires. Mais on peut aller plus loin 
en renonçant à valoriser et en assurant le contrôle d'efficience sur la base d'indicateurs physiques relativement aisés et peu coûteux à obtenir (Amans et Rascol-Boutard, 2008 ; Fabre, 2005). Au global, ce dernier constat explique sans doute l'essor des tableaux de bord opérationnels en collectivité constaté par plusieurs auteurs (Cour des comptes, 2009; Beaulier et Salery, 2006).

\subsection{Proposition d'une grille de questionnement}

En conclusion et à la lumière des développements précédents, l'élu ou le fonctionnaire territorial désirant disposer d'informations sur la consommation de ressources en collectivité, n'est pas tenu de suivre un chemin unique. Au contraire, il lui appartient de se livrer à un questionnement approprié de façon à déterminer les options les plus pertinentes compte tenu des objectifs impartis et des différentes contraintes opérationnelles, financières et politiques. Le tableau suivant recense les questions essentielles à résoudre ainsi que les diligences à effectuer.

Tableau 3 : Les grandes options en matière de calcul de coût

\begin{tabular}{|c|c|}
\hline Options à prendre et choix effectuables & Exigences essentielles \\
\hline 1) Faut-il un système exhaustif et homogène? & $\begin{array}{l}\text { Déterminer si l'utilité à long terme du système } \\
\text { justifie l'investissement }\end{array}$ \\
\hline 11) Système exhaustif et homogène & $\begin{array}{l}\text { Obtenir le visa du politique en prenant en compte } \\
\text { ses intérêts propres et ses contraintes }\end{array}$ \\
\hline 12) Ciblage sur certains services & $\begin{array}{l}\text { Sélectionner les services les plus pertinents } \\
\text { en fonction des problèmes rencontrés ou du } \\
\text { volontariat }\end{array}$ \\
\hline $\begin{array}{l}\text { 2) Faut-il un système permanent avec calculs de } \\
\text { coûts réguliers? }\end{array}$ & $\begin{array}{l}\text { Vérifier l'intérêt d'un suivi régulier des coûts } \\
\text { par rapport à l'objectif et aux moyens engagés }\end{array}$ \\
\hline 21) Système permanent & $\begin{array}{l}\text { Mettre en place un système permanent de collecte } \\
\text { et de traitement de l'information }\end{array}$ \\
\hline 22) Calculs de coûts ponctuels & Choisir une périodicité de réactualisation adaptée \\
\hline 3) Faut-il un calcul de coût complet? & $\begin{array}{l}\text { Examiner la pertinence de la prise en compte } \\
\text { des charges de structure, eu égard à l'objectif } \\
\text { imparti }\end{array}$ \\
\hline 31) Coûts complets & $\begin{array}{l}\text { Choisir une ventilation + ou - fine selon le degré } \\
\text { de précision requis }\end{array}$ \\
\hline 32) Coûts directs & Chiffrer également les prestations internes \\
\hline 4) Faut-il même un calcul de coût? & $\begin{array}{l}\text { Vérifier le caractère + ou - fixe des coûts ainsi que } \\
\text { l'étendue des variables d'action sur ceux-ci }\end{array}$ \\
\hline 41) Calculs de coûts & $\begin{array}{l}\text { Choisir la méthodologie adaptée en fonction des } \\
\text { exigences essentielles }\end{array}$ \\
\hline $\begin{array}{l}\text { 42) Absence de coûts et utilisation de mesures } \\
\text { physiques de la consommation de ressources }\end{array}$ & $\begin{array}{l}\text { Choisir un indicateur physique pertinent au niveau } \\
\text { de la consommation de ressources }\end{array}$ \\
\hline
\end{tabular}

Source : les auteurs 


\section{Conclusion}

Notre travail éclaire les résultats paradoxaux d'autres recherches qui s'inscrivent, elles aussi, dans des démarches de recherche action, qu'elles renvoient à des échecs ou à des succès. Il permet par exemple de comprendre pourquoi l'implantation d'un outil de gestion s'arrête net, alors que toutes les conditions paraissaient réunies pour un succès, du fait de l'absence de prise en compte de la dimension politique par les promoteurs du projet (Dreveton, 2008). Il conforte les résultats obtenus par d'autres chercheurs comme par exemple Rocher (2008) qui montre comment la transformation progressive du contenu du concept de consolidation des comptes est une nécessité pour que l'outil puisse se mettre en place.

Plus généralement, les apports de la déconstruction de cet idéal-type de la comptabilité de gestion nous semblent se situer à plusieurs niveaux. Au plan managérial, notre démarche peut aider les responsables dans les collectivités territoriales à développer des démarches plus appropriées, notamment en dialoguant de manière plus efficace avec des consultants, pour leur permettre d'élaborer des solutions au plus près des besoins de ces collectivités. Ces cadres territoriaux devraient ainsi être mieux à même de définir leurs besoins et le cahier des charges qui en découle. En outre, notre recherche pourrait éviter à d'autres organisations publiques, qui se lancent aujourd'hui dans des démarches d'implantation de systèmes de calcul des coûts, comme par exemple les universités (Portal et al., 2012), de s'engager dans des impasses (Ducrocq et Gervais, 2012). Au plan pédagogique, les enseignants pourraient proposer à leurs étudiants une vision plus réaliste de la comptabilité de gestion et donc leur donner les moyens d'une réflexion critique sur les outils qu'ils seront amenés à développer dans leur vie professionnelle ultérieure. Au plan théorique, la mise en évidence de cet idéal-type implicite nous semble en lui-même un résultat qui pourrait être généralisé à d'autres outils, que ce soit en contrôle de gestion (par exemple le tableau de bord) ou dans d'autres disciplines (par exemple en gestion des ressources humaines la gestion prévisionnelle des emplois et compétences). Plus largement, notre recherche apporte une contribution à la question de l'appropriation des outils de gestion (Moisdon, 1997; De Vaujany, 2006). Il faut toutefois constater également les nombreuses incertitudes persistantes. Les constatations faites sur le terrain tant par les professionnels que par les enseignants-chercheurs ainsi que les hypothèses faites sur le comportement des acteurs territoriaux face à l'outil analytique nécessitent, à notre sens, pour être pleinement confirmées, des études empiriques larges au niveau des services des mairies. On touche là aux limites bien connues de l'approche inductive (illustrées par la célèbre parabole de la dinde inductiviste de Russel). Celle-ci ouvre des portes et permet de faire émerger des hypothèses mais ne donne pas de réelles certitudes. 


\section{Bibliographie}

ALLÈGRE H., MOUTERDE F., (1991). Le contrôle de gestion. Éditions du moniteur, Paris.

AMANS P., RASCOL-BOUTARD S., (2008). La performance entre construit social et indicateur simplifié. Finance, Contrôle, Stratégie 11 (3), 45-63.

BARGAIN A., (2011). Histoire d'un outil de contrôle de gestion dans une collectivité locale : le cas de la comptabilité analytique à la ville d'Angers (1983-2005). XVI Journées d'Histoire de la Comptabilité et du Management, Nantes.

BARGAIN A., (2012). Retour sur un échec : le cas de la comptabilité analytique à la commune de La Rochesur-Yon (1988-1992). 33 Congrès de l'Association Francophone de Comptabilité, Grenoble.

BEAULIER M., SALERY Y., (2006). 20 ans de contrôle de gestion dans les collectivités locales : bilan et perspectives. Revue Française de Finances Publiques 95, 69-85.

BOUINOT J., (1977). La nouvelle gestion municipale - Comptabilité et management d'une commune. Cujas, Paris.

CAMEL E., (1987). Les managers à l'assaut des mairies. Sciences et Vie Économie 31, 56-59

CELLIER F., CHATELAIN-PONROY S. (2005). Les objectifs de performance et l'objectivité de la notion de performance. Workshop VM La performance publique locale; composants et mesures, disponible sur http:// www.ville-management.org.

CHAPET J.-M., (2007). Le système de gestion des collectivités territoriales : entre performance et délibération. Politiques et management public 25 (4), 1-19.

COENEN-HUTHER J., (2003). Le type idéal comme instrument de la recherche sociologique. Revue Française de Sociologie 4, 531-547.

COUR DES COMPTES (2009). Les évolutions du pilotage et du contrôle de la gestion des collectivités locales. In Cour des comptes. Rapport public annuel, 281-313.

DAVID A. (2008). La recherche-intervention, cadre général pour la recherche en management? In A. DAVID, A. HATCHUEL et R. LAUFER. Les nouvelles fondations des sciences de gestion $2^{\mathrm{e}}$ éd. FNEGE-Vuibert, Paris, 193-213.

DEMEESTÈRE R., (2000). Que peut-on attendre d'une comptabilité de gestion dans le secteur public. Politiques et management public 18 (4), 19-46.

DEMEESTÈRE R., (2007). L'analyse des coûts : public et privé. Politiques et management public 25 (3), $101-114$.

DE VAUJANY F.-X., (2006). Pour une théorie de l'appropriation des outils de gestion : vers un dépassement de l'opposition conception-usage. Management \& Avenir 3 (9), 109-126.

DREVETON B., (2008). Le rôle des représentations sociales au cours du processus de construction d'un outil de contrôle de gestion. Comptabilité Contrôle Audit 14 (2), 125-154.

DUCROCQ C, GERVAIS M., (2012). Les calculs de coûts complets par diplôme à l'université : quelle signification? 33 Congrès de 1'Association Francophone de Comptabilité, Grenoble.

DUPUIS J., (1991). Le contrôle de gestion dans les organisations publiques, PUF Paris.

FABRE P., (2005). Le contrôle des associations par les mairies françaises. Étude comparative portant sur les secteurs sport, culture et prévention de la délinquance. Doctorat en sciences de gestion. Université d'Orléans, Orléans.

FABRE P., BESSIRE D.,(2008). Les apports d'une recherche-intervention en collectivité territoriale : l'exemple de l'implantation d'une comptabilité de gestion dans une ville de taille moyenne, Congrès des IAE, 11-12 sept, Lille.

FEITZ A., (1990). Comptabilité analytique : quand la gestion débouche sur l'absurde. $S$ \& V Économie 62, 66-70.

GIBERT P. (1995). La difficile émergence du contrôle de gestion territorial. Politiques et management public 13 (3), 203-224.

GIBERT P., (2007). L'analyse des coûts dans le public : un équilibre impossible? Journée d'étude de l'institut de management public.

GIBERT P., THOENIG J-C., (1993). La gestion publique entre l'apprentissage et l'amnésie. Politiques et management public 11 (1), 3-21. 
HOFSTEDE G., (1981). Management Control of Public and not-for-profit Activities. Accounting, Organizations and Society 6 (3), 193-211.

HOOD CH., (1991). A Public Management for all Seasons? Public Administration 69, 3-19.

HOOD CH., (1995). The «New Public Management» in the 1980s: Variations on a Theme. Accounting, Organizations and Society 20 (2-3), 93-109.

LAWRENCE P.R., LORSCH J.W., (1974). Adapter les structures de l'entreprise. Les Éditions d'Organisation, Paris.

MEYSSONNIER F. (1993). Le contrôle de gestion communal : bilan et perspectives. Doctorat en Sciences de Gestion, Nancy : Université de Nancy 2.

MINISTÈRE DES COLLECTIVITÉS LOCALES, (1988). La comptabilité analytique appliquée aux services communaux. La Documentation Française, Paris.

MINTZBERG H., (1982). Structure et dynamique des organisations. Les Éditions d'Organisation, Paris.

MOISDON J.-CL., (1997). Du mode d'existence des outils de gestion. Seli-Arslan, Paris.

NIKITIN M., (1992). La naissance de la comptabilité industrielle en France. Doctorat en sciences de gestion, Université Paris Dauphine, Paris

PORTAL M., DREVETON B. et LANDE E., (2012). Construire un outil de comptabilité de gestion au sein d'une université - Retour sur un acte manqué. Management et Avenir 54, 126-144.

REY J.-P., (1991). Le contrôle de gestion des services publics communaux. Dunod CNFPT, Paris.

REY J.-P., (1994). Implanter le contrôle de gestion. Au-delà de la boîte à outils... La lettre du cadre territorial, Voiron.

ROCHER S., (2008). De l'implantation à l'appropriation d'un outil de gestion dans le secteur public local : une approche interactionniste. Comptabilité Contrôle Audit 14 (1), 49-68.

ROUSSARIE O.,(1994). Les outils de contrôle de gestion utilisés dans les services publics urbains - observation et interprétation des pratiques. Doctorat en Sciences de Gestion, Université de Poitiers, Poitiers.

SANDRETTO M.J., (1985). Comptabilité des coûts, quel est le meilleur système? Harvard - l'Expansion été, 82-94. WEBER M., (1965). Essai sur la théorie de la science (1904-1917). Plon, Paris. 


\section{Annexe : Méthodologie de la recherche}

L'article tente de restituer le cheminement intellectuel des auteurs dans le cadre d'une recherche non programmée de type inductif. Il faut ici en rappeler les trois phases (qu'on retrouve à un niveau plus général dans la première partie de l'article) :

Une première phase «d'application naturelle » des postulats de l'idéal-type sans que celui-ci soit formellement identifié en tant que tel mais avec l'appréhension claire de difficultés de mise en œuvre.

$\Rightarrow>$ À ce niveau, la formation et l'expérience antérieure importante acquise dans le domaine de la comptabilité de gestion et plus particulièrement pour ce qui est de son implantation en collectivité ont constitué le soubassement intellectuel de la recherche.

Une deuxième phase de constatation de l'inadéquation des postulats de l'idéal-type avec un début de représentation de celui-ci mais de façon fragmentaire.

=> À ce niveau, la méthodologie a été du domaine de la recherche intervention avec deux contrats de recherche portant sur la mise en place de comptabilités de gestion dans deux villes de taille moyenne (2006 et 2011 pour la mise en œuvre opérationnelle). Les contraintes importantes en font des situations limites qui permettent de tester la robustesse de l'outil et la pertinence des concepts sous-jacents. Les enseignements tirés de l'expérience de ces deux recherches-interventions ont été confirmés lors d'un séminaire d'experts (2007) qui a permis de confronter les différents points de vue à ce sujet.

Une troisième phase de mise en évidence de l'ensemble des postulats de l'idéal-type, l'inadéquation de ces postulats aux contraintes des collectivités servant de cadre explicatif aux difficultés récurrentes d'implantation de la comptabilité de gestion.

=> À ce niveau, il a fallu mener une recherche documentaire de type « historique » en s'appuyant sur les archives vu l'ancienneté (relative) de certaines sources (annales des examens comptables; premiers ouvrages sur la comptabilité analytique dans les communes...). 